PACS 72.10.-d, 72.20.-I, 72.20.Fr

\title{
Features of piezoresistance in heavily doped n-silicon crystals
}

\author{
G.P. Gaidar \\ Institute for Nuclear Research, National Academy of Sciences of Ukraine, \\ 47, prospect Nauky,03680 Kyiv,Ukraine; e-mail: gaydar@kinr.kiev.ua
}

\begin{abstract}
It has been shown that in silicon single crystals heavily doped with arsenic the presence of the temperature gradient at the interface of the liquid and solid phases in the process of growing them from a melt does not lead to anisotropy of piezoresistance under the passing current both along the direction of deforming load $(J\|X\|\langle 111\rangle)$ and perpendicularly to it $(J \perp X \|\langle 111\rangle)$. This is considered as an evidence of the dominant influence of randomization in spatial distribution of a dopant due to $k T$ (at $T=1685 \mathrm{~K}$ ) during the growth of single crystals.
\end{abstract}

Keywords: silicon, heavily doped single crystals, piezoresistance, scattering.

Manuscript received 26.10.12; revised version received 03.12.12; accepted for publication 26.01.13; published online 28.02.13.

\section{Introduction}

Semiconductor is considered to be heavily doped if the Bohr radius of impurity states $\left(a_{B}\right)$ exceeds the average distance between the impurity centers $\left(\sim N_{I}^{-\frac{1}{3}}\right)$, i. e. when

$a_{B}>N_{I}^{-\frac{1}{3}}$ or $a_{B}^{3} N_{I}>1$,

where $a_{B}=\frac{4 \pi \varepsilon_{0} \hbar^{2}}{m^{*} e^{2}} ; \varepsilon_{0}$ is the permittivity of vacuum; $\hbar=h / 2 \pi, h-$ Planck constant; $m^{*}$ - effective electron mass; $e$ - electron charge. In the case of elemental semiconductors, the criterion (1) corresponds to a sufficiently narrow interval of the dopant concentrations, even in the assumption that the lower bound of heavy doping is defined by the condition $a_{B}^{3} N_{I} \approx 1$, and the given in [1] values of the upper bound coincide with the solubility limit for dopants in the corresponding crystals.

With increasing the impurity concentration, semiconductor passes into the degenerate state, when the Fermi level $\left(E_{\mathrm{F}}\right)$ gets into the allowed band. In this case, the region near $E_{\mathrm{F}}$ becomes actual for the kinetic effects, because it is the area where the most essential changes in the state of charge carriers occur under the action of external influences.

Non-uniform distribution of impurities within the crystal bulk leads to the local fluctuations of their concentration and, as a result, the potential energy of carriers is not quite periodic. The random field of impurities, which represents a set of chaotically placed humps (wells) of arbitrary height (depth) and shapes, bends the boundaries of the allowed energy bands. As a result, a certain density of electron states ("tails" of the density of states) arises in the energy region corresponding to the forbidden band of undoped semiconductor and exponentially [2] attenuates (decreases) when deepening into the forbidden band. These are the so-called fluctuating levels, which are located in the potential wells [3].

These levels remain discrete even at high concentrations of impurity centers. This is one of the specific peculiarities of these levels, which was confirmed by the experiments with photo- and electroluminescence in heavily doped semiconductors [4]. That is why, in such single crystals, along with the region of quasi-continuous values of the energy (in the allowed bands), the discrete spectrum regions (which can be densely occupied) appear at the boundaries of the allowed bands. 
In the most used temperature range $(77 \ldots 300) \mathrm{K}$ and with slight doping semiconductors such as $\mathrm{Si}$ and $\mathrm{Ge}$, the contribution from scattering by acoustic phonons and ions of dopant impurities, as a rule, prevails over other possible mechanisms of scattering. With increasing of doping level, according to [5], the assumption about the individual nature of scattering of electrons by each of ions cannot be applied. Indeed, non-uniform distribution of impurities in the semiconductor bulk leads possibly to aggregation of the impurity atoms, which is influenced by the crystal growth. Under this condition, electron scattering occurs not by individual ions, but by their aggregates. Since the scattering probability is proportional to the square of the scattering center charge, this should lead to a significant reduction in the mobility of carriers.

In relatively weakly doped $n$-Si at $T=77 \mathrm{~K}$ (all the more at higher temperatures), the impurity centers are completely ionized. It is known [6] that at a low concentration of the impurity atoms, a shift of the conduction band edge with applying uniaxial elastic deformation results in the corresponding energy shift of the impurity center state without changing the ionization energy that is counted from the bottom of the conduction band. The change of the ionization energy in this case can be caused only by the change in the effective masses with deformation. But this effect is quite insignificant and can appear not always, but only conditioned by shift deformation.

The situation changes significantly in the case of heavily doped $n$-Si single crystals, when the increase in the impurity atom concentration leads to the overlapping of their wave functions and to the appearance of the impurity band. It is known [7] that the ionization energy of phosphorus impurity in $n-\mathrm{Si} \quad(\approx 0.045 \mathrm{eV})$ is approximately 4 times higher than the value of this parameter for $n-\mathrm{Ge}(\approx 0.012 \mathrm{eV})$. As a result of this (ceteris paribus), complete ionization of donor centers in the moderately and heavily doped (but nondegenerate) $n$ - $\mathrm{Si}$ crystals (in contrast to $n-\mathrm{Ge}$ ) even at room temperature is not achieved [8]. Consequently, uniaxial elastic deformation can influence on the total concentration of charge carriers in the conduction band (c-band) due to changes of the depth of donor levels $\Delta E_{d}$ related with the different (non-equivalent) minima of the energy. The change in the overlapping degree of the wave functions of impurity centers under the action of uniaxial elastic deformation also influences on the activation energy of impurity centers [9].

\section{Results and discussion}

Piezoresistance and piezo-Hall effect [10] as well as piezoresistance in the uniaxially deformed (at $J\|X\|\langle 001\rangle) n$-Si crystals highly doped with various impurities of the donor type (P, Sb, As) [11] were studied. The authors of these works paid attention that the effect of the charge carrier scattering on the fluctuations in impurity concentrations is essentially revealed not only in the conditions of measurement of the maximal resistance changes when the electric current $J$ passes along the axis of deformation (i. e. at $J\|X\|\langle 001\rangle)$, but also in the conditions when the electric current $J$ is oriented perpendicularly to this axis (i. e. at $J \perp X \|\langle 001\rangle$ ) [12]. The authors [13] focused on the fact that both the theory [6] and the cyclotron resonance data in the uniaxially deformed $n$-Si [14] show that in the presence of the component of deformation shift (typical for $X \|\langle 111\rangle$ in $n$-Si), the transverse effective mass of charge carriers $\left(m_{\perp}^{*}\right)$ increases linearly with the growth of $X$ approximately by $1 \%$ for every $0.25 \mathrm{GPa}$.

In consideration of the general expression for the mobility of charge carriers in the many-valley semiconductors

$\mu=\frac{e}{3} \frac{\left\langle\tau_{\perp}\right\rangle}{m_{\perp}^{*}}\left(2+\frac{1}{K}\right) \approx \frac{2}{3} e \frac{\left\langle\tau_{\perp}\right\rangle}{m_{\perp}^{*}}$,

where, according to [15],

$\tau_{\perp}=\frac{a_{\perp}}{T \sqrt{\varepsilon}}, a_{\perp}=\frac{\pi c_{11}^{\prime} \hbar^{4}}{k \mathrm{C}_{1}^{2} \sqrt{2 m_{\|} m_{\perp}^{2}}} \frac{1}{\Phi_{1 a}}=\frac{A}{m_{\perp}^{*}}$.

at $A=$ const, since the $\Phi_{1 a}$ function very weakly depends on the longitudinal $\left(m_{\|}^{*}\right)$ and transverse $\left(m_{\perp}^{*}\right)$ effective masses, the direct coupling of sufficiently specific piezoresistance in $n-\mathrm{Si}$ (at $J\|X\|\langle 111\rangle$ ) with the mobility of charge carriers $(\mu)$ becomes apparent. Indeed, the comparison of (2) with (3) shows that

$\mu \sim \frac{1}{m_{\perp}^{* 2}}$

Consequently, in the conditions of $J\|X\|\langle 111\rangle$, piezoresistance arises in $n-\mathrm{Si}$ only due to the deformation growth of $m_{\perp}^{*}(X)$ (and reduction of the mobility $\mu$ ), when in the conduction band the carrier concentration is completely constant $\left(n_{e}=\right.$ const) and their interminimum redistribution is absent.

At the sufficiently high doping level of $n$-Si, which is accompanied by the occurrence of density-ofstates tails, and under deformation of the crystal in the direction $X \|\langle 111\rangle$, the piezoresistance related with the change of $n_{e}$ in $c$-band can arise. Indeed, it was earlier shown [16] that under deformation of $n-\mathrm{Si}$ (at $X \|\langle 111\rangle)$, the energy gap $\Delta E_{d}$ between the bottom of the conduction band and level of impurities increases with the growth of $X$, but does not decrease, as it occurs at $X \|\langle 001\rangle$ or $X \|\langle 110\rangle$. And in the conditions of partial ionization of the impurity centers, at $T=$ const this will result in the growth of piezoresistance owing to deformation carrier freezing-out from the conduction band. 
In elemental semiconductors (such as $\mathrm{Si}$ and $\mathrm{Ge}$ ), the elements of III and V groups of the Periodic Table are hydrogen-like impurities, which are completely ionized in $\mathrm{Ge}$ at temperatures above $20 \mathrm{~K}$, and in $\mathrm{Si}-$ above $50 \mathrm{~K}$. Since the ground-state energy for the hydrogen-like center is defined as follows $E_{0}=-\frac{m^{*} e^{4}}{8 h^{2} \varepsilon_{0}^{2}}$, it is possible that the physical basis of the $\Delta E_{d}$ increase with the growth of $X \|\langle 111\rangle$ is the deformation increase of $m_{\perp}^{*}$ in the presence of the deformation shift $[6,14]$. Similarly, as was shown in [13], in weakly doped $n$-Si the increase of $m_{\perp}^{*}$ with $X$ provides enhancement of the piezoresistance, starting with the smallest mechanical stresses of the compression $X\|J\|\langle 111\rangle$. This effect, in contrast to the traditional Smith-Herring piezoresistance $[17,18]$, is characterized by the fact that in the investigated range $X(0 \ldots 1.5 \mathrm{GPa})$ satisfies the inequality

$$
\frac{\rho_{X}^{\langle 111\rangle}}{\rho_{0}}(300 \mathrm{~K})>\frac{\rho_{X}^{\langle 111\rangle}}{\rho_{0}}(77 \mathrm{~K}),
$$

even at the transition from pure to moderately $(0.5<\rho<500 \mathrm{Ohm} \cdot \mathrm{cm})$ doped crystals (in moderately doped crystals the impurity level is expanded up to the impurity band, but between the latter and the conduction band the energy gap still remains). However, the experiments [16] carried out with using rather heavily doped $n$-Si crystals $\left(\rho_{300 \mathrm{~K}} \approx 0.02 \mathrm{Ohm} \cdot \mathrm{cm}\right)$ confirmed the inequality (5) only in the limited region of the mechanical stresses $X \leq 0.7 \mathrm{GPa}$ (see Fig. 1, region $A$ ). At the same time, with a further increase of $X>(0.7 \ldots 0.8) \mathrm{GPa}$ the sign inversion will take place as a result of deformation freezing-out of charge carriers from the conduction band (see the inequality (5) and Fig. 1, region $B$ ).

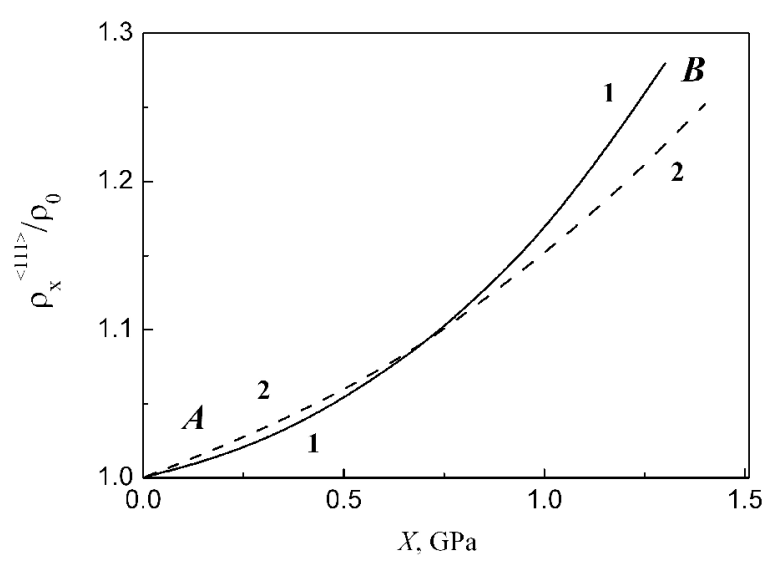

Fig. 1. Dependences $\rho_{\mathrm{x}} / \rho_{0}=f(X)$ measured in heavily doped $n-\mathrm{Si} \quad\left(\rho_{300 \mathrm{~K}} \approx 0.02 \mathrm{Ohm} \cdot \mathrm{cm}\right)$ under the condition $J\|X\|\langle 111\rangle$ at different temperatures $T: 1-77 \mathrm{~K}, 2-300 \mathrm{~K}$.

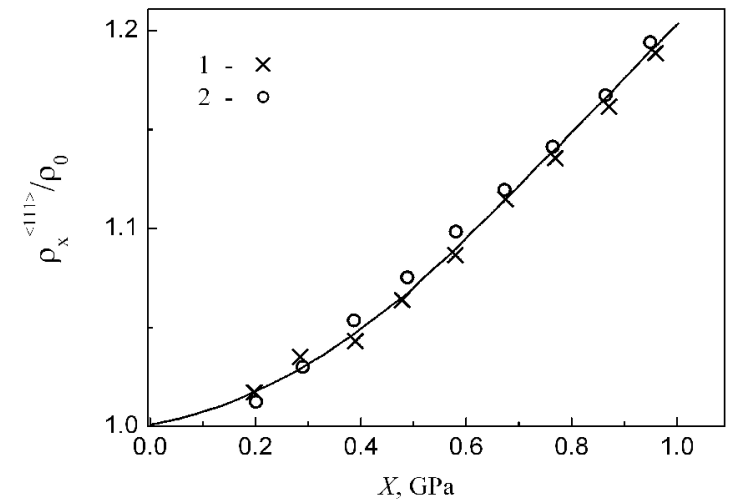

Fig. 2. Piezoresistance (at $T=4.2 \mathrm{~K}$ ) of heavily doped $n-\mathrm{Si}\langle\mathrm{As}\rangle\left(\rho_{300 \mathrm{~K}} \approx 0.0043 \mathrm{Ohm} \cdot \mathrm{cm}\right)$ under the passing current directed in the different ways regarding to the crystal growth axis coinciding with the crystallographic direction $\langle 111\rangle: 1-$ $J\|X\|\langle 111\rangle ; 2-J \perp X \|\langle 111\rangle$.

The determination of the piezoresistance nature at the symmetric disposition of the deformation axis relatively to the isoenergetic ellipsoids in $n$ - $\mathrm{Si}$ (in the conditions of carrying out the measurements at $J\|X\|\langle 111\rangle$ ) did not give yet the answer to the following question: can the temperature gradient, existing at the interface of the solid and liquid phases during the growth of single crystals, lead to tangible anisotropy of the piezoresistance with respect to the crystallization front that coincides with the plane (111)? The answer to this question could be obtained only as a result of comparison of the measurements carried out on the samples cut along the crystallographically equivalent directions $\langle 111\rangle$ : first sample - along the direction in which the crystal was grown, and the second sample is located with respect to the first one at the angle $72^{\circ}$. There are no reasons to expect a significant effect of anisotropy associated with the presence of the crystallization front due to the sufficiently high melting temperature of the silicon crystals $\left(T_{\text {melt }} \sim 1412^{\circ} \mathrm{C}\right)$. However, a possibility to detect this effect of anisotropy (certainly, in case of its existence) would be real, using the relative values not even for $\rho_{x} / \rho_{0}$, but for the corresponding voltages $V_{\mathrm{x}} / V_{0}$, which significantly increases the accuracy of the experiments.

For the experiments concerning detection of abovediscussed anisotropy, $n-\mathrm{Si}\langle\mathrm{As}\rangle$ with $n_{e 300 \mathrm{~K}} \approx$ $1.07 \cdot 10^{17} \mathrm{~cm}^{-3}$ grown in the crystallographic direction $\langle 111\rangle$ by the Czochralski method was used. The samples were cut in the corresponding directions with the accuracy of determining the orientation (using the conventional X-ray method) not worse than $15^{\prime}$. The measurements of piezoresistance were carried out under the passing current both along the deforming load direction $X$ and perpendicularly to it, i. e. in the conditions $J\|X\|\langle 111\rangle$ and $J \perp X \|\langle 111\rangle$. The results obtained at $4.2 \mathrm{~K}$ (presented in Fig. 2) indicate a lack of 
anisotropy of "technological origin", although the above-discussed piezoresistance nonrelated with the interminimum redistribution of charge carriers (i. e. this piezoresistance is not the traditional one by SmithHerring), within the limits of mechanical loads that we used $0 \leq X \leq 1.0 \mathrm{GPa}$ (starting with $X \geq 0.2 \mathrm{GPa}$ ), was measured quite reliably.

\section{Conclusions}

As a result of the performed experiments, it has been found that in the $n$-type silicon samples heavily doped with arsenic, anisotropy of piezoresistance (and consequently, the electrical conductivity and spatial distribution of the fluctuating aggregates of impurity) is absent under the passing current both along the deforming load $(J\|X\|\langle 111\rangle)$ and perpendicularly to it $(J \perp X \|\langle 111\rangle)$. This conclusion (within the limits of the reached accuracy of the experiment) can be considered as reliable, since the measurements of $\rho_{X}^{\langle 111\rangle} / \rho_{0}$ were made under the passing current in those directions (relatively to the direction of the crystal growth), in which the effect of anisotropy (in case of its existence) should appear the most distinctly.

\section{References}

1. V.I. Fistul', Heavily Doped Semiconductors. Nauka, Moscow, 1967 (in Russian).

2. B.I. Shklovskii, A.L. Efros, The tails of density of states in heavily doped semiconductors // Fizika $i$ tekhnika poluprovodnikov, 4 (2), p. 305-316 (1970), in Russian.

3. A.L. Efros, Density of states and interband light absorption in heavily doped semiconductors // Uspekhi fizicheskikh nauk 3 (3), p. 451-482 (1973), in Russian.

4. A.P. Levaniuk, V.V. Osipov, The luminescence theory of heavily doped semiconductors // Fizika $i$ tekhnika poluprovodnikov, 7 (6), p. 1056-1068 (1973), in Russian.

5. I.V. Dakhovskii, T.A. Polyanskaya, A.G. Samoilovich, Yu.V. Shmartsev, Concerning the mobility of electrons in heavily doped semiconductors // Fizika i tekhnika poluprovodnikov, 4 (11), p. 2165 2171 (1970), in Russian.

6. G.L. Bir, G.E. Pikus, Symmetry and Deformation Effects in Semiconductors. Nauka, Moscow, 1972 (in Russian).
7. R. Smith, Semiconductors. Mir, Moscow, 1982 (in Russian).

8. S.S. Li, W.R. Thurder, The dopant density and temperature dependence of electron mobility in ntype silicon // Solid State Electron. 20(7), p. 609616 (1977).

9. A.E. Belyaev, R.M. Vinetsky, O.P. Gorodnichii, Determination of the deformation potential of shallow donors in silicon // Solid State Communs. 44 (3), p. 403-406 (1982).

10. J. Kinoshita, Piezoresistance and piezo-Hall effect in heavily doped n-type silicon // J. Phys. Soc. Japan. 33 (3), p. 743-746 (1972).

11. P.I. Baranskii, V.V. Kolomoets, Yu.A. Okhrimenko, Piezoresistance and Hall effect in heavily doped crystals of n-Si // Fizika i tekhnika poluprovodnikov, 19 (10), p. 1768-1770 (1985), in Russian.

12. P.I. Baranskii, V.V. Kolomoets, Yu.A. Okhrimenko, Piezoresistance of degenerated crystals of $n-\mathrm{Si}$ along and across to the axis of deformation // Fizika i tekhnika poluprovodnikov, 19 (8), p. 1411 1413 (1985), in Russian.

13. P.I. Baranskii, V.V. Kolomoets, A.V. Fedosov, Piezoresistance arising in conditions of symmetric arrangement of deformation axis relatively to all isoenergetic ellipsoids in $n$ - $\mathrm{Si} / /$ Fizika i tekhnika poluprovodnikov, 13 (4), p. 815-819 (1979), in Russian.

14. J.C. Hensel, H. Hasegawa, M. Nakayama, Cyclotron resonance in uniaxially stressed silicon. II. Nature of the covalent bond // Phys. Rev. 138(1A), p. A225-A238 (1965).

15. P.I. Baranskii, I.S. Buda, I.V. Dakhovskii, V.V. Kolomoets. Electrical and Galvanomagnetic Phenomena in Anisotropic Semiconductors (Ed. by P.I. Baranskii). Naukova Dumka, Kiev, 1977 (in Russian).

16. P.I. Baranskii, V.V. Kolomoets, Yu.A. Okhrimenko, A.V. Fedosov, Influence of uniaxial elastic deformation on the ionization energy of phosphorus impurity in silicon crystals // Fizika i tekhnika poluprovodnikov $16(2)$, p. 361-364 (1982), in Russian.

17. G. S. Smith, Piezoresistance effect in germanium and silicon // Phys. Rev. 94 (1), p. $42-49$ (1954).

18. C. Herring, Transport properties of a many-valley semiconductor // Bell System Techn. J. 34 (2), p. $237-290$ (1955). 